\title{
Decomposition in diverse dimensions
}

\author{
Eric Sharpe \\ Department of Physics \\ Robeson Hall, 0435 \\ 850 Drillfield Drive \\ Blacksburg, VA 24061 \\ ersharpe@vt.edu
}

This paper discusses the relationships between gauge theories defined by gauge groups with finite trivially-acting centers, and theories with restrictions on nonperturbative sectors, in two and four dimensions. In two dimensions, these notions seem to coincide. Generalizing old results on orbifolds and abelian gauge theories, we propose a decomposition of two-dimensional nonabelian gauge theories with center-invariant matter into disjoint sums of theories with rotating discrete theta angles; for example, schematically, $S U(2)=S O(3)_{+}+S O(3)_{-}$. We verify that decomposition directly in pure nonsupersymmetric two-dimensional Yang-Mills as well as in supersymmetric theories. In four dimensions, by contrast, these notions do not coincide. To clarify the relationship, we discuss theories obtained by restricting nonperturbative sectors. These theories violate cluster decomposition, but we illustrate how they may at least in special cases be understood as disjoint sums of well-behaved quantum field theories, and how dyon spectra can be used to distinguish, for example, an $S O(3)$ theory with a restriction on instantons from an $S U(2)$ theory. We also briefly discuss how coupling various analogues of Dijkgraaf-Witten theory, as part of a description of instanton restriction via coupling TQFT's to QFT's, may modify these results. 


\section{Contents}

1 Introduction

2 Two dimensional gauge theories

2.1 Review of orbifolds and abelian gauge theories . . . . . . . . . . 6

2.2 Discrete theta angles . . . . . . . . . . . . . . . . . . . 9

2.3 Decomposition conjecture for nonabelian gauge theories . . . . . . . . . 11

2.4 Decomposition in nonsupersymmetric pure gauge theories . . . . . . . . . 13

2.5 Decomposition in theories with center-invariant matter . . . . . . . . 19

2.6 Decomposition of supersymmetric theories . . . . . . . . . . . 20

3 Four dimensional theories $\quad 25$

3.1 Dyon charge lattices . . . . . . . . . . . . . . . 26

3.2 Vafa-Witten TFT partition functions . . . . . . . . . . . . . 29

4 Dijkgraaf-Witten theory

5 Conclusions $\quad 32$

6 Acknowledgements

A Miscellaneous cocharacter lattice relations

\begin{tabular}{ll|} 
References & 35 \\
\hline
\end{tabular} 


\section{Introduction}

This paper concerns the relationship between gauge theories in which the gauge group has a center that acts trivially on the matter, and theories with restrictions on instantons, and applies the relationship to generate some identities for gauge theories in various dimensions.

In more detail, a $G$ gauge theory where $G$ is semisimple with center $C$ will typically have fewer instantons than a corresponding $G / C$ gauge theory. A prototype is the relationship between $S U(2)$ and $S O(3)$ gauge theories; the latter contain instantons that do not lift to $S U(2)$.

Now, although an $S U(2)$ gauge theory contains fewer instantons, it need not be the same as an $S O(3)$ theory with a restriction on instantons. The latter, by a well-known result of Weinberg, violates cluster decomposition, whereas an $S U(2)$ gauge theory by itself will not, at least in four dimensions.

Thus, we have two related but in general distinct notions:

- A gauge theory with a trivially-acting subgroup,

- A gauge theory with a restriction on instantons.

In the special case of two dimensions, we will argue that these notions coincide. This is a natural generalization of previous work on orbifolds and abelian gauge theories, encapsulated in the 'decomposition conjecture' formulated in [1]. For orbifolds of the form $[X / \Gamma]$, for example, for a finite group $\Gamma$, this says that if a nontrivial subgroup of $\Gamma$ acts trivially on the theory, the CFT of the orbifold is equivalent to a disjoint union of CFT's for effective orbifolds. Projection operators onto the various components are formed from linear combinations of the dimension-zero twist fields and the identity operator.

Since there are no gauge dynamics in two dimensions, one would expect analogous behaviors in nonabelian gauge theories, and that is what we argue here. Specifically, for a $G$ gauge theory, $G$ semisimple, with $K$ a finite subgroup of the center, if the matter of the theory is invariant under $K$, then we argue that the theory decomposes as a disjoint union of $G / K$ gauge theories with variable discrete theta angles. For example, schematically,

$$
S U(2)=S O(3)_{+}+S O(3)_{-}
$$

This can be explicitly checked in a number of examples. For one, we describe how Migdal's exact solution of two-dimensional pure Yang-Mills decomposes as above, as well as how the exact partition functions of supersymmetric gauge theories on $S^{2}$ also decompose (when they have center-invariant matter). 
Phrased another way, we will argue in this paper that the decomposition conjecture of [1], which does not specify presentations, does indeed extend to two-dimensional nonabelian gauge theories, as expected.

In four dimensions, these notions are distinct. An $S U(2)$ gauge theory is simply not the same as a sum of two $S O(3)$ theories, for example. One can still enforce a restriction on instantons in an $S O(3)$ theory, but the resulting physical theory differs from an $S U(2)$ theory. We will discuss how to see this distinction explicitly in the spectrum of e.g. dyons in the theory.

The first half of this paper, section 2, is devoted to studying examples of two-dimensional gauge theories. We begin with a review of the application of the decomposition conjecture to orbifolds and abelian gauge theories, which describes how existence of a trivially-acting finite subgroup of the orbifold or gauge group implies that the theory 'decomposes' into a disjoint union of mutually non-interacting ordinary theories. As gauge fields in two dimensions do not have propagating degrees of freedom, one would expect the same behavior in nonabelian gauge theories with trivially-acting finite subgroups, which we check in examples. The precise statement of decomposition for nonabelian gauge theories utilizes two-dimensional discrete theta angles, so we review those before discussing general cases. We then discuss pure nonsupersymmetric two-dimensional Yang-Mills as a prototype, and observe how Hilbert spaces, partition functions, and Wilson line vevs factorize in precisely the fashion indicated by decomposition. We also discuss how decomposition can be seen in theories with centerinvariant matter, first via Higgsing to abelian gauge theories, and then by examining exact expressions for supersymmetric partition functions on $S^{2}$.

In section 3 we turn to four dimensional theories. Here, gauge theories with triviallyacting finite subgroups are not equivalent to gauge theories with restrictions on nonperturbative sectors. For example, the latter will violate cluster decomposition whereas the former need not, unlike two-dimensional cases. It has been argued that four dimensional SCFT's with restrictions on instantons may obey an analogue of the decomposition conjecture, decomposing into a disjoint union of theories, though much less work has been done in four dimensions than two. To better understand both four dimensional decomposition and the distinction above, we examine dyon spectra in theories with restrictions on nonperturbative sectors, and observe how they can be used to distinguish, for example, an $S U(2)$ gauge theory from an $S O(3)$ gauge theory with a restriction on instantons obeying an analogue of decomposition. We also discuss Vafa-Witten topological field theory partition functions in theories with a restriction on instantons, which seem to provide a simple example of four dimensional decomposition in action.

Finally, in section 4 we discuss Dijkgraaf-Witten theories, as they are sometimes described in the literature alongside certain constructions of theories with restrictions on instantons. Coupling to analogues of Dijkgraaf-Witten theory modifies (sometimes, altogether removes) the decomposition property that much of this paper is devoted to, as previously observed in 
two dimensions in [1]. We briefly review Dijkgraaf-Witten theory in this context and outline its effects.

Recently there has been a tremendous amount of progress in understanding two-dimensional supersymmetric gauge theories and gauged linear sigma models, one of the central tools used to study string compactifications, and dualities therein (see for example [2 6] for a few recent contributions). It is our hope that the results presented here on two-dimensional nonabelian gauge theories will be useful to that effort.

\section{Two dimensional gauge theories}

Briefly, in two-dimensional theories, we claim that a gauge theory with a trivially-acting finite subgroup is equivalent to a disjoint union of theories with effectively-acting gauge groups, unlike four dimensional gauge theories.

For orbifolds and abelian gauge theories in two dimensions, this phenomenon has by now been extensively documented, and also used to make predictions for Gromov-Witten invariants, predictions which have since been checked. In this context the result has been known as the 'decomposition conjecture' [1].

Because in two dimensions gauge fields have no propagating degrees of freedom and hence no gauge dynamics, surely the same decomposition conjecture that applies to orbifolds and abelian gauge theories must also apply to two-dimensional nonabelian gauge theories. Briefly, if the center of the gauge group acts trivially on the massless matter, then the theory contains a trivially-acting orbifold, and the analysis for orbifolds implies that the theory should decompose.

We begin by reviewing the application of the decomposition conjecture to two-dimensional orbifolds and abelian gauge theories, and then turn to nonabelian gauge theories in two dimensions, where we will see analogous phenomena. The different factors appearing in the decomposition will have different discrete theta angles, so we then review of discrete theta angles, and formulate the general decomposition claim for nonabelian gauge theories in two dimensions. We check the decomposition conjecture in detail for pure nonsupersymmetric Yang-Mills theory in two dimensions, then discuss checks when center-invariant matter is present, and finally check via partition functions for $(2,2)$ supersymmetric theories (obtained via localization). 


\subsection{Review of orbifolds and abelian gauge theories}

For two-dimensional orbifolds and abelian gauge theories, it is by now well-understood that existence of a trivially-acting subgroup is physically equivalent to a restriction on nonperturbative sectors. Such theories break 1 cluster decomposition, but in a very mild fashion which is remedied by the observation that the theory decomposes into a disjoint union of physical theories with no mutual interactions, indexed by irreducible representations of the trivially-acting subgroup. This has been extensively discussed and numerous examples described in e.g. [1,7-17]. (See also [18 20] for reviews, and 21-26] for checks of applications to Gromov-Witten theory).

This behavior is very different from four dimensions: there, an $S U(2)$ gauge theory has instantons that are a subset of those of an $S O(3)$ gauge theory, but the $S U(2)$ gauge theory does not itself necessarily decompose into a disjoint union of physical theories. We will explore such relationships in four dimensions in section 3 .

As this behavior in two dimensions has been extensively discussed elsewhere, in this section we will merely give a brief overview of the highlights, in order to make this paper somewhat self-contained.

Perhaps the simplest example of this phenomenon is provided by an orbifold in which one quotients a trivial group action on a space. As a trivial example, consider $\left[X / \mathbb{Z}_{n}\right]$ where the $\mathbb{Z}_{n}$ acts trivially on the entire space. In this case, all of the points on $X$ are fixed under the orbifold group action, and the usual prescription 2 yields a total of $n-1$ dimension zero twist fields and a spectrum consisting of $n$ copies of the cohomology of $X$. As this spectrum contains multiple dimension zero operators, it violates cluster decomposition, but in the mildest possible way: this spectrum is equivalent to that of a theory on a disjoint union of $n$ copies of $X$. In that description, projection operators onto the various components of the disjoint union are formed from discrete Fourier transforms of the dimension zero operators, both in this and more general orbifolds of this form. As a result, correlation functions factorize, both in this and more general orbifolds of this form.

A less trivial example is given by the orbifold $\left[X / D_{4}\right]$, where $D_{4}$ is the eight-element group with center $\mathbb{Z}_{2}$. The elements are given by

$$
\{1, z, a, b, a z, b z, a b, b a=a b z\},
$$

where $z$ generates the center of $D_{4}$. The quotient $D_{4} / \mathbb{Z}_{2}$ is $\mathbb{Z}_{2} \times \mathbb{Z}_{2}$, whose elements we will

\footnotetext{
1 In passing, note that cluster decomposition and locality are different concepts. For example, [11] [appendix B] shows how two-dimensional BF theory at level $k>1$, which is manifestly local, violates cluster decomposition.

2 The reader might well be concerned that the usual prescription breaks down in such a case. However, it was shown in e.g. 7 that for example modular invariance requires the inclusion of all twisted sectors of this form in general.
} 
represent as

$$
\{1, \bar{a}, \bar{b}, \overline{a b}\},
$$

where, for example, $\bar{a}$ represents the coset $\{a, a z\}$. Now, the one-loop partition function of the $D_{4}$ orbifold is of the form

$$
Z\left(D_{4}\right)=\frac{1}{\left|D_{4}\right|} \sum_{g, h g h=h g} Z_{g, h},
$$

where $Z_{g, h}$ denotes the contribution from sectors with boundary conditions determined by the commuting pair $(g, h)$. Now, as the $\mathbb{Z}_{2}$ acts trivially on $X$, for each pair $(g, h)$ that appears in the orbifold, their contribution matches that of a $\mathbb{Z}_{2} \times \mathbb{Z}_{2}$ orbifold:

$$
Z_{g, h}=Z_{\bar{g}, \bar{h}}
$$

However, not every commuting pair in $\mathbb{Z}_{2} \times \mathbb{Z}_{2}$ lifts to a commuting pair in $D_{4}$ - as expected on general principles, the $D_{4}$ orbifold has fewer nonperturbative sectors (i.e. twisted sectors) than the $\mathbb{Z}_{2} \times \mathbb{Z}_{2}$ orbifold. Specifically, there are no $D_{4}$ twisted sectors of the form

$$
a \underset{b}{a}, \quad a \square_{a b}, \quad b \underset{a b}{\square},
$$

as the pairs $(a, b),(a, a b),(b, a b)$ do not commute in $D_{4}$. (However, the omitted sectors close under $S L(2, \mathbb{Z})$, so there is no violation of modular invariance.) Thus, first, the $D_{4}$ orbifold cannot match the $\mathbb{Z}_{2} \times \mathbb{Z}_{2}$ orbifold, as the partition function of the former is missing some of the sectors of the latter. Furthermore, the sectors that are missing, are the same as the ones that acquire signs when one turns on discrete torsion in a $\mathbb{Z}_{2} \times \mathbb{Z}_{2}$ orbifold. In fact, with a little work, one can show

$$
Z\left(D_{4}\right)=Z\left(\mathbb{Z}_{2} \times \mathbb{Z}_{2}\right)+Z\left(\mathbb{Z}_{2} \times \mathbb{Z}_{2}, \text { with discrete torsion }\right),
$$

the essential point being that the problematic sectors cancel out and so do not appear in the $D_{4}$ orbifold.

In open strings, this decomposition can be seen more directly on the open string states. A subgroup of the orbifold group that acts trivially on the target space can act nontrivially on the Chan-Paton factors. However, just from taking invariants, the only contributions that can arise in the open string spectrum are from open strings connecting Chan-Paton factors in the same representation of the subgroup in question. In this way one quickly sees a natural decomposition of the theory, indexed by irreducible representations of the pertinent subgroup, with no open string interactions between different sectors.

So far we have discussed orbifolds, but analogous results also hold in abelian gauge theories in two dimensions with massless states of nonminimal charge. One prototype for such considerations is an analogue of the ordinary $(2,2)$ supersymmetric $\mathbb{C P}^{N-1}$ model, with gauge group $U(1)$ and $N$ chiral superfields of charge $k>1$, instead of charge 1 (so that 
after Higgsing, a $\mathbb{Z}_{k}$ survives to act, trivially, on the fields). Now, perturbatively, minimal and nonminimal charges are indistinguishable - one can rescale one to get the other - but nonperturbatively, the resulting theories can be different [7]:

- On a compact worldsheet, to uniquely specify the matter fields, one must specify a vector bundle to which they couple. The invariant meaning of different charge assignments is in terms of different bundles, and different bundles imply different zero modes, hence different anomalies, and so different physics.

- On a noncompact worldsheet, a $U(1)$ gauge theory with minimal charges can be distinguished from one with nonminimal charges by the existence of massive minimallycharged fields. Even if their masses are above the cutoff scale in the theory, their presence can still be detected via periodicity of the two-dimensional $\theta$ angle.

Such abelian gauge theory examples containing a trivially-acting finite subgroup also obey decomposition. As a concrete example, consider a generalization of the $\mathbb{C} \mathbb{P}^{N-1}$ example above, a GLSM with $N$ chiral superfields $x_{i}$, another chiral superfield $z$, and gauge group $U(1)^{2}$ with charges

\begin{tabular}{cc}
$x_{i}$ & $z$ \\
\hline 1 & $-n$ \\
0 & $k$
\end{tabular}

where $n$ is positive. This theory has D-terms

$$
\begin{aligned}
\sum_{i}\left|x_{i}\right|^{2}-n|z|^{2} & =r_{1} \\
k|z|^{2} & =r_{2},
\end{aligned}
$$

where we assume $r_{1} \gg 0, r_{2} \gg 0$. This example is discussed in [9] [section 3.3]. The second $U(1)$ nearly gauges away the $z$, except for a remaining trivial $\mathbb{Z}_{k}$ action. The remaining fields and $U(1)$ describe the $\mathbb{C} P^{N-1}$ model. The result 3 is $\mathbb{C P}^{N-1}$ with a trivial $\mathbb{Z}_{k}$ action, classified by $n$ mod $k$. The quantum cohomology ring of this theory was shown in [9] [section 3.3] to be

$$
\mathbb{C}[x, y] /\left(x^{N} y^{-n}=q, y^{k}=1\right) .
$$

The different values of $y$ index $k$ different copies of the quantum cohomology ring of $\mathbb{C P}^{N-1}$, with shifting $B$ fields. Thus, the quantum cohomology ring of this theory is consistent with decomposition.

\footnotetext{
${ }^{3}$ Technically, this is the GLSM for a $\mathbb{Z}_{k}$ gerbe on $\mathbb{C P}^{N-1}$, of characteristic class $-n \bmod k \in$ $H^{2}\left(\mathbb{C P}^{N-1}, \mathbb{Z}_{k}\right)$.
} 
The considerations above also apply to nonlinear sigma models in two dimensions. If we restrict nonperturbative sectors, the resulting theory violates cluster decomposition, but in an extremely mild way, as the result is equivalent to a disjoint union of ordinary theories with variable $B$ fields. Schematically, we can see this in the path integral for a nonlinear sigma model as follows. To project onto sectors with worldsheet instantons defined by maps $\phi$ such that

$$
\int \phi^{*} \omega
$$

is divisible by $n$, for some fixed two-form $\omega$, for example, is accomplished by inserting a projection operator of the form

$$
\int[D \phi] e^{-S}\left(\sum_{k=0}^{n-1} e^{2 \pi i(k / n) \int \phi^{*} \omega}\right),
$$

which is the same as

$$
\sum_{k=0}^{n-1} \int[D \phi] \exp \left(-S+2 \pi i(k / n) \int \phi^{*} \omega\right) .
$$

In this second form, the interpretation as a disjoint union of theories with rotating $B$ fields (defined by $(k / n) \omega)$ is clear.

The decomposition conjecture [1] gives a very precise description of how to build the disjoint union from the original nonlinear sigma model with a restriction on instantons. Such theories also give physical realizations of strings on certain stacks known as gerbes, and so the decomposition conjecture makes physical predictions for Gromov-Witten invariants of strings on gerbes. These predictions have been rigorously proven, see for example [21 26].

\subsection{Discrete theta angles}

Just as in four dimensions [27, 28], there is also a discrete theta angle in two dimensions, as has been observed in [16, 29 31]. These discrete theta angles play an essential role in the form of decomposition for two-dimensional nonabelian gauge theories, so we review them here.

Consider a $G$ gauge theory with $G=\tilde{G} / K, \tilde{G}$ semisimple and simply-connected, and $K$ a finite subgroup of the center of $\tilde{G}$. $K$ will be a product of cyclic groups, i.e.

$$
K=\mathbb{Z}_{n_{1}} \oplus \mathbb{Z}_{n_{2}} \oplus \cdots
$$

There is a degree two $K$-valued characteristic class $w$, which for $S O(n)$ for example would be the degree two Stiefel-Whitney class. Each such characteristic class can be used to define 
a discrete theta angle via a term in the action which is schematically ${ }^{4}$ of the form

$$
\theta^{\prime} \int w
$$

for $\theta^{\prime}$ a character 5 of $K$.

Thus, for example, in two dimensions there are two $S O(3)$ gauge theories, which we shall label $S O(3)_{ \pm}$, just as in four dimensions.

In general, the set of discrete theta angles for a gauge group $G$ as above is indexed by the set $\hat{K}$ of irreducible representations of $K$ (which, since $K$ is a finite abelian group, has as many elements as $K$, and since $K$ is a sum of cyclic groups, can be identified with the characters of $K$ ). Thus, we will index discrete theta angles in $G$ gauge theories by $\sqrt{6} \mu \in \hat{K}$.

Furthermore, if $G$ is any semisimple group and $K$ a finite subgroup of its center, then a $G / K$ gauge theory will contain $|K|=|\hat{K}|$ more discrete theta angles than $G$. These new discrete theta angles will not be entirely independent of the old ones. For example, $\left(S U(4) / \mathbb{Z}_{2}\right) / \mathbb{Z}_{2}=S U(4) / \mathbb{Z}_{4}$ has two more discrete theta angles relative to $S U(4) / \mathbb{Z}_{2}$, which itself has two, so that $S U(4) / \mathbb{Z}_{4}$ has four altogether. More generally, letting $C$ denote centers, if $G=\tilde{G} / K$ then there is an extension

$$
1 \longrightarrow K \longrightarrow C(\tilde{G}) \longrightarrow C(G) \longrightarrow 1
$$

and hence the discrete theta angles are related by 7

$$
1 \longrightarrow \widehat{C(G)} \longrightarrow \widehat{C(\tilde{G})} \longrightarrow \hat{K} \longrightarrow 1
$$

\footnotetext{
${ }^{4}$ To be clear, such finite-group-valued cohomology does not have a representation in de Rham cohomology, so we are using an integral loosely to denote a formal contraction.

${ }^{5}$ As $w$ is $K$-valued, one contracts with a character of $K$ to derive a number.

${ }^{6}$ As a minor consistency check, note that the components of the decomposition in orbifolds and abelian gauge theories described in [1] were also indexed by irreducible representations. Here, this is a consequence of the construction of discrete theta angles, whereas in [1] the justification was completely different, relying instead on e.g. observations on open string spectra.

7 A few details may be useful for some readers. The dual sequence above is obtained by applying $\operatorname{Hom}(-, U(1))$, and so one gets a sequence

$$
1 \longrightarrow \widehat{C(G)} \longrightarrow \widehat{C(\tilde{G})} \longrightarrow \hat{K} \longrightarrow \operatorname{Ext}^{1}(C(G), U(1))
$$

In the cases at hand, $C(G)$ will always be a sum of cyclic groups, and $\operatorname{Ext}^{1}\left(\mathbb{Z}_{n}, U(1)\right)=0$, hence $\operatorname{Ext}^{1}(C(G), U(1))=0$. To see this, we apply $\operatorname{Hom}(-, U(1))$ to the sequence$$
0 \longrightarrow \mathbb{Z} \stackrel{\times n}{\longrightarrow} \mathbb{Z} \longrightarrow \mathbb{Z}_{n} \longrightarrow 0
$$

to get

$$
\operatorname{Hom}(\mathbb{Z}, U(1)) \longrightarrow \operatorname{Hom}(\mathbb{Z}, U(1)) \longrightarrow \operatorname{Ext}^{1}\left(\mathbb{Z}_{n}, U(1)\right) \longrightarrow 0
$$

and use $\operatorname{Hom}(\mathbb{Z}, U(1))=U(1)$, hence the first map is the surjective $n$th power map and $\operatorname{Ext}^{1}\left(\mathbb{Z}_{n}, U(1)\right)=0$. We would like to thank S. Katz for the computation shown here.
} 


\subsection{Decomposition conjecture for nonabelian gauge theories}

Briefly, if a nonabelian gauge theory with semisimple gauge group $G$ has a center $C(G)$ that acts trivially on the massless matter of the theory, then this theory implicitly contains a trivially-acting $C(G)$ orbifold, and so ought to admit the same decomposition structure described earlier. We shall check this assertion in detail, utilizing exact solutions for pure nonsupersymmetric Yang-Mills theory and recent localization-derived exact results for supersymmetric gauge theories in two dimensions.

To set notation, let $\tilde{G}$ denote a semisimple, simply-connected, compact Lie group. We will compare $\tilde{G}$ gauge theories with center-invariant matter to $\tilde{G} / C(\tilde{G})$ gauge theories with the same matter, where $C(\tilde{G})$ denotes the center. The latter theories have (finitely many) discrete theta angles, indexed by irreducible representations of $C(\tilde{G})$. Letting a theory with discrete theta angle indexed by $\mu \in \widehat{C(\tilde{G})}$ be denoted

$$
(\tilde{G} / C(\tilde{G}))_{\mu}
$$

we will argue that the gauge theories decompose as

$$
\tilde{G}=\sum_{\mu \in \widetilde{C(\tilde{G})}}(\tilde{G} / C(\tilde{G}))_{\mu} .
$$

For example,

$$
S U(2)=S O(3)_{+}+S O(3)_{-}
$$

and

$$
S U(4)=\left(S U(4) / \mathbb{Z}_{4}\right)_{0}+\left(S U(4) / \mathbb{Z}_{4}\right)_{1}+\left(S U(4) / \mathbb{Z}_{4}\right)_{2}+\left(S U(4) / \mathbb{Z}_{4}\right)_{3} .
$$

A little more generally, for any semisimple compact $G$, not necessarily simply-connected, and for $K$ a subgroup of the center, we have discrete theta angles in $\hat{K}$ and a decomposition

$$
G=\sum_{\lambda \in \hat{K}}(G / K)_{\lambda}
$$

Now, in this case, $G$ itself may also have discrete theta angles, so we can generalize to include the decomposition of theories with discrete theta angles.

Generalizing along such lines, let $G=\tilde{G} / K$ for $\tilde{G}$ as above and $K$ some subgroup of $C(\tilde{G})$, where $G$ has center $C(G)$. Then, $G$ gauge theories are indexed by a discrete theta angle $\lambda \in \hat{K}$. In this case, we can decompose $G$ gauge theories with discrete theta angle $\lambda$ as

$$
(G)_{\lambda}=\sum_{\mu \in \widehat{C(G)}}(G / C(G))_{\lambda, \mu} .
$$


Since

$$
G / C(G)=\tilde{G} / C(\tilde{G})
$$

we can interpret $(\lambda \in \hat{K}, \mu \in \widehat{C(G)})$ as defining an element 8 of $\widehat{C(\tilde{G})}$. To be somewhat more specific, we will choose always an isomorphism that respects the extension (11), by which we mean, if $g \in C(\tilde{G})$ is in the image of $K$, then we define $(\lambda, \mu)$ to be such that

$$
(\lambda, \mu)(g)=\lambda(g) .
$$

As a consequence, these decompositions are related as follows:

$$
\tilde{G}=\sum_{\lambda \in \hat{K}}(G)_{\lambda}=\sum_{\rho \in \widetilde{C(\tilde{G})}}(\tilde{G} / C(\tilde{G}))_{\rho} .
$$

We list a few examples of the predictions of this more general form of the decomposition conjecture below. First, using the fact that

$$
S O(4)=\frac{S U(2) \times S U(2)}{\mathbb{Z}_{2}}
$$

we have

$$
\begin{aligned}
& S O(4)_{+}=S O(3)_{+} \times S O(3)_{+}+S O(3)_{-} \times S O(3)_{-} \\
& S O(4)_{-}=S O(3)_{+} \times S O(3)_{-}+S O(3)_{-} \times S O(3)_{+} .
\end{aligned}
$$

Note as a consequence that

$$
\begin{aligned}
S U(2) \times S U(2) & =S O(4)_{+}+S O(4)_{-}, \\
& =\sum_{i, j= \pm} S O(3)_{i} \times S O(3)_{j},
\end{aligned}
$$

as expected from equation (7).

Another example follows from the fact that the center of $S U(4)$ is $\mathbb{Z}_{4}$. If instead we take a $\mathbb{Z}_{2}$ quotient, then we get

$$
\begin{aligned}
& \left(S U(4) / \mathbb{Z}_{2}\right)_{+}=\left(S U(4) / \mathbb{Z}_{4}\right)_{0}+\left(S U(4) / \mathbb{Z}_{4}\right)_{2}, \\
& \left(S U(4) / \mathbb{Z}_{2}\right)_{-}=\left(S U(4) / \mathbb{Z}_{4}\right)_{1}+\left(S U(4) / \mathbb{Z}_{4}\right)_{3} .
\end{aligned}
$$

\footnotetext{
8 There are noncanonical isomorphisms of sets $\hat{K} \times \widehat{C(G)} \rightarrow \widehat{C(\tilde{G})}$. Unless $\widehat{C(\tilde{G})}$ splits, however, the product structure will be more complicated.
} 
The result above follows from the fact that $\mathbb{Z}_{4} / \mathbb{Z}_{2}$ contains two cosets, $\{0,2\}$ and $\{1,3\}$. Note that, as a consequence,

$$
\begin{aligned}
S U(4) & =\left(S U(4) / \mathbb{Z}_{2}\right)_{+}+\left(S U(4) / \mathbb{Z}_{2}\right)_{-}, \\
& =\sum_{k=0}^{3}\left(S U(4) / \mathbb{Z}_{4}\right)_{k},
\end{aligned}
$$

as expected from equation (7).

\subsection{Decomposition in nonsupersymmetric pure gauge theories}

In this section we will verify the decomposition claim for nonsupersymmetric pure Yang-Mills theories. For theories with vanishing discrete theta angles, exact expressions for Hilbert spaces, partition functions, and so forth are known (see for example [32 36]). To verify the decomposition claim, we will utilize extensions of those results to theories with discrete theta angles. Such extensions have been discussed previously in [29], and we will discuss and elaborate on them here9.

Let us begin with a discussion of the Hilbert spaces of nonsupersymmetric pure Yang-Mills theory with gauge group $G=\tilde{G} / K$, where $\tilde{G}$ is compact, semisimple, and simply-connected, and $K$ is a (finite) subgroup of the center of $\tilde{G}$.

For the theory with vanishing discrete theta angles, the conventional gauge theory, the Hilbert space is the space of functions $f(g)$ on $G$, invariant under conjugation. Such functions, known as class functions, can be expanded in an analogue of a Fourier series in characters $\chi$ of $G$, as

$$
f(g)=\sum_{R} c_{R} \chi_{R}(g)
$$

for constants $c_{R}$ determined by the function $f$.

Now, let us consider the Hilbert space of the corresponding theory with a nonzero discrete theta angle defined by $\lambda \in \hat{K}$. Because of the discrete theta angle term in the Lagrangian, a particle moving around a closed noncontractible path must pick up a phase. Thus, we should think about the Hilbert space as consisting of sections of a line bundle on $G$, or equivalently a class function $f$ on $\tilde{G}$ satisfying

$$
f(g z)=\lambda(z) f(g)
$$

where $z \in K$. As a result, $f(g)$ can be expanded in terms of characters $\chi_{R}$ of $\tilde{G}$ which are in a fixed representation of $K$.

\footnotetext{
${ }^{9}$ We would like to thank Y. Tachikawa for discussions of his results on two-dimensional nonsupersymmetric pure Yang-Mills theories with discrete theta angles.
} 
Before going on to describe partition functions, let us briefly describe how the result above is compatible with decomposition. Recall from equation (4) that decomposition predicts that for pure nonsupersymmetric Yang-Mills theories with semisimple gauge group $G$ and $K$ a (finite) subgroup of the center, the theory should decompose in a fashion we indicate schematically as

$$
G=\sum_{\lambda \in \hat{K}}(G / K)_{\lambda}
$$

Now, the Hilbert space of the $G$ gauge theory contains all class functions on $G$. On the lefthand-side, the Hilbert space of each $(G / K)_{\lambda}$ theory contains class functions of $G$ which are in a fixed representation of $K$. The set of all class functions on $G$ has a natural decomposition according to representations of $K$, and so we see that the Hilbert spaces match in exactly the fashion predicted by decomposition.

In addition, as $G$ gauge theories may themselves have discrete theta angles, decomposition makes a more refined conjecture (5),

$$
(G)_{\lambda}=\sum_{\mu \in \widehat{C(G)}}(G / C(G))_{\lambda, \mu}
$$

for $G=\tilde{G} / K, K$ a subgroup of $C(\tilde{G})$. In terms of the Hilbert space under discussion, this reflects the fact since $K$ commutes with $C(\tilde{G})$, class functions on $\tilde{G}$ are simultaneously representations of $K$ and $C(\tilde{G})$. In particular, the Hilbert space on the left, consisting of class functions in representation $\lambda$ of $K$, can be further decomposed according to representations of $C(\tilde{G})$.

A brief example may help illuminate this matter. Suppose $\tilde{G}=S U(4)$ and $K=\mathbb{Z}_{2}$, so $G=S U(4) / \mathbb{Z}_{2}$. The Hilbert space of a pure $G$ gauge theory of the form discussed here then decomposes into class functions of fixed $\mathbb{Z}_{2}$ representations. However, each $\mathbb{Z}_{2}$ representation can be further decomposed into $\mathbb{Z}_{4}$ representations. If we let $n \in\{0,1,2,3\}$ characterize representations of $\mathbb{Z}_{4}$, then, schematically,

$$
\begin{array}{ccc}
\text { trivial rep' of } \mathbb{Z}_{2} & \leftrightarrow & 0,2 \text { of } \mathbb{Z}_{4} \\
\text { nontrivial rep' of } \mathbb{Z}_{2} & \leftrightarrow & 1,3 \text { of } \mathbb{Z}_{4}
\end{array}
$$

In this fashion, we see that Hilbert spaces reproduce the decomposition (5).

Now, let us turn to partition functions. In two-dimensional pure gauge theories with vanishing discrete theta angles, the partition functions are known exactly [32 34], and are of the form ( [35] [equ'n (3.20)], [36] [equ'n (2.51)])

$$
Z=\sum_{R}(\operatorname{dim} R)^{2-2 g} \exp \left(-A C_{2}(R)\right)
$$


where $g$ is the genus of the two-dimensional spacetime, $A$ its area, and the sum is over representations of the gauge group.

Now, let us work out partition functions of two-dimensional theories with discrete theta angles. To that end, it is helpful to consider a genus-one surface with one end sliced off, as illustrated below:

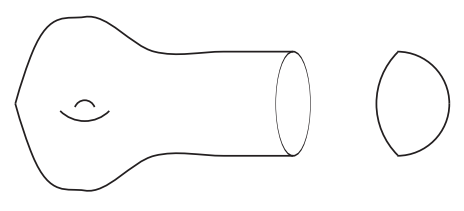

Since the wavefunctions around noncontractible loops are, as discussed above, understood as sections of nontrivial bundles over $G$, the partition function of the left slice must also be such a section. Since the entire partition function is obtained by gluing, and the contribution of the left-hand-side is a section of a nontrivial bundle, the contribution from the cap on the right must also be a section of the same nontrivial bundle.

Such sections of nontrivial bundles can be understood in terms of class functions on $\tilde{G}$ associated to specific $\mu \in \hat{K}$, as previously discussed. The resulting partition function, for a theory with discrete theta angle defined by $\mu \in \hat{K}$, should be of the same form as before, namely

$$
Z=\sum_{R}(\operatorname{dim} R)^{2-2 g} \exp \left(-A C_{2}(R)\right)
$$

except that here, the sum over representations is restricted to representations $R$ of $G$ associated with $\mu$. (This result has previously been given in [29].)

The result above is invariant under retriangulations, by virtue of nearly identical computations to cases with vanishing discrete theta angles. The essential point is that to prove independence from choice of triangulation and related results requires the following four identities [35, 36]:

$$
\begin{gathered}
\int d U \overline{\chi_{R}(U)} \chi_{R^{\prime}}(U)=\delta_{R, R^{\prime}}, \\
\sum_{R}\left|\chi_{R}\right\rangle\left\langle\chi_{R}\right|=1 \\
\int d U \chi_{R}\left(A U B U^{-1}\right)=\frac{1}{\operatorname{dim} R} \chi_{R}(A) \chi_{R}(B), \\
\int d V \chi_{R}(A V) \chi_{R^{\prime}}\left(V^{-1} B\right)=\delta_{R, R^{\prime}} \frac{1}{\operatorname{dim} R} \chi_{R}(A B) .
\end{gathered}
$$


Three of the equations above apply automatically without modification. Only equation (9) requires any thought. Because $K$ is a subgroup of the center, $K$ commutes with the gauge group, and so the sum over representations in equation (9) can be further diagonalized to provide a set of completeness relations, one for each representation of $K$. Thus, after suitable renormalizations, one can write

$$
\sum_{R, \text { fixed } \mu}\left|\chi_{R}\right\rangle\left\langle\chi_{R}\right|=1
$$

for each $\mu \in \hat{K}$.

We can derive results for partition functions axiomatically, as in [35] [section 3.7], from a nearly identical ansatz:

$$
\begin{aligned}
\text { Cap } & =\sum_{R}(\operatorname{dim} R) \chi_{R}(U) e^{-A C_{2}(R)} \equiv Z_{\text {cap }}(U), \\
\text { Tube } & =\sum_{R}|R\rangle\langle R| e^{-A C_{2}(R)}, \\
\text { Pants } & =\sum_{R}|R\rangle \otimes|R\rangle \otimes|R\rangle \frac{e^{-A C_{2}(R)}}{\operatorname{dim} R},
\end{aligned}
$$

where in each case, the sum is over representations associated to fixed $\mu \in \hat{K}$.

In this language, we can understand invariance under retriangulations, for example, in the following standard fashion. Consider a cap which has been subdivided down the middle, as illustrated below:

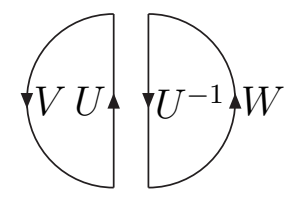

To be independent of triangulation means that if we glue the caps above along $U$, the result should be a cap in which $U$ does not appear, i.e.

$$
\int d U Z_{\text {cap }}(V U) Z_{\text {cap }}\left(U^{-1} W\right)=Z_{\text {cap }}(V W)
$$

and this is a nearly immediate consequence of equation (11). This analysis is nearly identical to that of [35] [section 3.4.2], the only difference being that here sums over representations are restricted to those representations with fixed $\mu \in \hat{K}$. Other arguments from [35] [chapter 3] apply here to two-dimensional Yang-Mills with discrete theta angles with equal immediacy. 
To make the discussion above more clear, let us outline the results for the $S U(2)$ and $S O(3)_{ \pm}$gauge theories. For $S U(2)$, the partition function is given by

$$
Z=\sum_{R}(\operatorname{dim} R)^{2-2 g} \exp \left(-A C_{2}(R)\right)
$$

where the sum is over all representations $R$ of $S U(2)$, and for $S O(3)_{+}$, the sum is over all representations of $S O(3)$. For $S O(3)_{-}$, the sum is over $S U(2)$ representations that are not also $S O(3)$ representations. That sounds somewhat odd as a description of an $S O(3)$ gauge theory, but the point is that it should be interpreted in terms of sections of nontrivial bundles over $S O(3)$.

In this case, decomposition should now be clear: the sum appearing in the $S U(2)$ gauge theory is the sum of the representations appearing in the $S O(3)_{+}$and $S O(3)_{-}$gauge theories, so the partition functions obey decomposition:

$$
Z(S U(2))=Z\left(S O(3)_{+}\right)+Z\left(S O(3)_{-}\right) .
$$

More generally, in a $G$ gauge theory, summing over the various discrete theta angles in $G / K$ gauge theories reproduces the sum over $G$ representations, and so the partition functions obey decomposition (4):

$$
Z(G)=\sum_{\lambda \in \hat{K}} Z\left((G / K)_{\lambda}\right) .
$$

It is not difficult to see how the further generalization (5) also arises. Recall this form of the decomposition conjecture says, schematically,

$$
(G)_{\lambda}=\sum_{\mu \in \widehat{C(G)}}(G / C(G))_{\lambda, \mu}
$$

for $G=\tilde{G} / K$. Here, the point is that the partition function

$$
Z\left((G)_{\lambda}\right)
$$

involves a sum over representations of $\tilde{G}$ that are in a fixed representation of $K$. As noted in the discussion of Hilbert spaces, representations of $\tilde{G}$ in a fixed representation of $K$ can be further decomposed into representations of $C(G)$, hence the partition functions decompose in the fashion outlined above.

In addition to exact expressions for Hilbert spaces and partition functions, there also exist exact expressions for vevs of Wilson lines in two-dimensional Yang-Mills, see for example [35] [section 3.5], 37] [equ'n (3)]. These expressions also generalize to nonzero discrete theta angles, and obey a decomposition principle. 
Briefly, closely following the discussion in [35] [section 3.5.1] for nonintersecting loops, if one has a Wilson loop defined by a curve $\Gamma$ and representation $R_{\Gamma}$,

$$
W\left(R_{\Gamma}, \Gamma\right)=\operatorname{Tr}_{R_{\Gamma}} P \exp \left(\oint_{\Gamma} A\right)
$$

then

$$
\left\langle W\left(R_{\Gamma}, \Gamma\right)\right\rangle=\int d U \prod_{c} Z\left(\Sigma_{c}, U, U^{-1}\right) W\left(R_{\Gamma}, \Gamma\right)
$$

where the $\Sigma_{c}$ are the various components of the two-dimensional spacetime obtained after slicing along $\Gamma$, with boundaries labelled by group elements $U, U^{-1}$ according to orientation. As in [35] [section 3.5.1], the expression above can be written as a sum over representations, in essentially the usual form.

From the discussion above, we see that vevs of Wilson lines factorize in exactly the same fashion as Hilbert spaces and partition functions, and for the same fundamental reason. The vev of a Wilson line in a $(G)_{\lambda}$ theory, for example, for $G=\tilde{G} / K$ and $\lambda \in \hat{K}$, is defined by a sum over $\tilde{G}$ representations associated to the fixed representation $\lambda$ of $K$. As before, those representations can be further decomposed into representations of $C(G)$, from which the usual decomposition statement follows. As the details are more or less identical to what has been described previously, we will not extensively elaborate. In passing, note that the vev of a given Wilson line in some components may vanish - the sum will add up to the vev of the Wilson line, but the contributions to the sum need not be separately nonzero.

Briefly, as one further consistency check, (q-deformed) pure nonsupersymmetric twodimensional Yang-Mills was related to $G / G$ gauged WZW models in [38]. We will not discuss gauged WZW models in great detail, but we do note in passing that they necessarily obey their own analogue of the decomposition conjecture. Specifically, if $G$ is a semisimple Lie group and $H \subset G$ a subgroup with center $C$, then in a $G / H$ gauged WZW model, since one gauges the adjoint action of $H$, the center $C$ necessarily acts trivially on the theory, so applying the same reasoning as for orbifolds and abelian gauge theories, the $G / H$ gauged WZW model must also obey decomposition.

As another consistency check, pure nonsupersymmetric two-dimensional Yang-Mills is also closely related to $B F$ theory in two dimensions, see for example [39]. It is therefore relevant to mention that $B F$ theory in two dimensions at level $k>1$ also exhibits a breakdown in cluster decomposition, which can be understood in terms of a decomposition of the theory into disjoint sectors [11][appendix B].

Finally, in passing, two-dimensional pure nonsupersymmetric Yang-Mills also arises in other contexts. One such is described in [36] [section 3.1], in terms of volumes of moduli spaces of flat connections. We will not elaborate on such matters here, but we do note that the decomposition of volumes of moduli spaces of flat $S O(3)$ connections described there is consistent with the decomposition conjecture presented here. 


\subsection{Decomposition in theories with center-invariant matter}

Let us now consider adding center-invariant matter, to a not-necessarily-supersymmetric pure gauge theory in two dimensions. We claim the decomposition conjecture continues to hold. We are not aware of exact partition function results for non-supersymmetric gauge theories with matter10, but we can perform other checks.

Specifically, we can Higgs the nonabelian gauge symmetry to a subgroup, and apply the decomposition conjecture in its form for orbifolds and abelian gauge theories to recover a decomposition of the desired form.

First, consider a two-dimensional $S U(2)$ theory containing an adjoint scalar. At a generic point on the Higgs branch, the $S U(2)$ has been Higgsed to $U(1)$. If the other matter is invariant under the $\mathbb{Z}_{2}$ center, then its $U(1)$ charges must be even, as $\{ \pm 1\} \subset U(1)$ must leave the matter invariant.

Now, we know from our review of abelian gauge theories that a $U(1)$ gauge theory with massless matter of even charges 11 will decompose into a disjoint union of two theories, with variable flat $B$ fields. We can understand each of those components as the result of Higgsing the two $S O(3)$ gauge theories. In this language, the flat $B$ fields are the low-energy description of the discrete theta angles.

For another example, consider an $S U(3)$ gauge theory with center-invariant matter, As above, consider a point on the Higgs branch where the $S U(3)$ is Higgsed to a $U(1)^{2}$ (so that we can apply our understanding of abelian gauge theories). Since the center of $S U(3)$ is generated by

$$
\operatorname{diag}(\xi, \xi, \xi)
$$

for $\xi$ a primitive third root of unity, and the $U(1)^{2} \subset S U(3)$ is given by

$$
\operatorname{diag}\left(e^{i \theta_{1}}, e^{i \theta_{2}}, e^{-i\left(\theta_{1}+\theta_{2}\right)}\right),
$$

we see that if the matter is invariant under the center of $S U(3)$, then it must be invariant under the subgroup $\left(1, \xi, \xi^{2}\right) \subset U(1)$ for each $U(1)$, and hence all matter fields must have, after Higgsing, charges that are multiples of three. At this point, as before, we can apply our understanding of decomposition in abelian gauge theories to argue that, at least along the Higgs branch, the theory decomposes into a disjoint union of three theories, each of which should be the result of Higgsing an $S U(3) / \mathbb{Z}_{3}$ gauge theory with suitable discrete theta angle. In this fashion we get another consistency check of the decomposition conjecture for nonabelian gauge theories.

\footnotetext{
${ }^{10}$ That said, there has been recent progress in solving two-dimensional Yang-Mills with adjoint-valued matter [40].

${ }^{11}$ In purely abelian gauge theories, one also needed to give massive minimally-charged matter, and then one could use $\theta$ angle periodicities to distinguish these theories from theories obtained merely by a rescaling. Here, equivalent information seems to be implicitly encoded in the fact that we are Higgsing an $S U(2)$ theory.
} 
More generally, given a $G=\tilde{G} / K$ gauge theory, $\tilde{G}$ semisimple, simply-connected, and compact, $K$ a subgroup of the center, with matter that is invariant under $K$, essentially the same considerations apply on the Higgs branch. Briefly, if we Higg 12 the gauge group $G$ to a product of $U(1)$ 's, and, because the matter is center-invariant, the $U(1)$ charges must all be nonminimal. The known version of the decomposition conjecture then applies to give results consistent with the nonabelian version described in section 2.3 .

\subsection{Decomposition of supersymmetric theories}

For $(2,2)$ supersymmetric gauge theories on $S^{2}$, there are now exact results for partition functions [41,42], obtained via localization. In this section, we will show that those exact partition functions for supersymmetric theories with center-invariant matter obey the decomposition conjecture stated previously in section 2.3. We will follow the notation of [41].

Let us begin with a comparison of the supersymmetric partition functions for the pure $S U(2)$ and $S O(3)_{ \pm}$theories. Following [41] [equ'n (3.34)], the partition function of a supersymmetric theory on $S^{2}$ is given by

$$
Z_{S^{2}}=\frac{1}{|\mathcal{W}|} \sum_{\mathfrak{m}} \int\left(\prod_{j} \frac{d \sigma_{j}}{2 \pi}\right) Z_{\text {class }}(\sigma, \mathfrak{m}) Z_{\text {gauge }}(\sigma, \mathfrak{m}) \prod_{\Phi} Z_{\Phi}(\sigma, \mathfrak{m} ; \tau, \mathfrak{n}),
$$

where [41] [equ'n (3.35)]

$$
\begin{aligned}
Z_{\text {class }}(\sigma, \mathfrak{m}) & =e^{-4 \pi i \xi \operatorname{Tr} \sigma-i \theta \operatorname{Tr} \mathfrak{m}} \exp (8 \pi i r \operatorname{Re} \tilde{W}(\sigma / r+i \mathfrak{m} /(2 r))) \\
Z_{\text {gauge }}(\sigma, \mathfrak{m}) & =\prod_{\alpha \in G}\left(\frac{|\alpha(\mathfrak{m})|}{2}+i \alpha(\sigma)\right)=\prod_{\alpha>0}\left(\frac{\alpha(\mathfrak{m})^{2}}{4}+\alpha(\sigma)^{2}\right) \\
Z_{\Phi}(\sigma, \mathfrak{m} ; \tau, \mathfrak{n}) & =\prod_{\rho \in R_{\Phi}} \frac{\Gamma\left(\frac{R[\Phi]}{2}-i \rho(\sigma)-i f^{a}[\Phi] \tau_{a}-\frac{\rho(\mathfrak{m})+f^{a}[\Phi] n_{a}}{2}\right)}{\Gamma\left(1-\frac{R[\Phi]}{2}+i \rho(\sigma)+i f^{a}[\Phi] \tau_{a}-\frac{\rho(\mathfrak{m})+f^{a}[\Phi] n_{a}}{2}\right)}
\end{aligned}
$$

The notation above follows [41]. Briefly, $f^{a}[\Phi]$ encodes the non-R-charges of a chiral multiplet $\Phi$, and $R[\Phi]$ its R-charge. $R_{\Phi}$ denotes the corresponding representation of the gauge group. $\mathcal{W}$ denotes the Weyl group of the gauge group. $\tau=\left(\tau_{a}\right)$ and $\mathfrak{n}=\left(n_{a}\right)$ define twisted masses for the chiral superfield.

The $\mathfrak{m}$ are elements of the Lie algebra of a Cartan subgroup of the torus, corresponding to elements of the cocharacter or dual weight 13 lattice for the gauge group, meaning for any

\footnotetext{
${ }^{12}$ If the matter is such that such a Higgsing is not possible, then it is not possible to reduce to considerations of abelian gauge theories and apply older results.

13 The term 'dual weight' lattice can be ambiguous: some authors occasionally use the term 'weight lattice'
} 
representation $R$ of the gauge group and corresponding weight $\rho, \rho(\mathfrak{m}) \in \mathbb{Z}$. In admittedly ambiguous notation, we will use $\mathfrak{m}$ to denote both pertinent Lie algebra elements as well as more abstract elements of the cocharacter lattice, indexed by tuples of integers.

For our purposes, it will be essential to understand how the sum over m's varies depending upon the precise group. Let us first compare the sum for $S U(2)$ and $S O(3)$. Briefly, the character lattice of $S U(2)$ is twice as large as the character lattice of $S O(3)$, since the oddspin representations are only representations of $S U(2)$. Since the character lattice of $S U(2)$ is larger, the cocharacter lattice of $S U(2)$ must be smaller. In particular, if we normalize such that for $S O(3), \mathfrak{m}$ varies over all integers, then for $S U(2), \mathfrak{m}$ must vary over only even integers.

Next, let us turn to discrete theta angles in this example. First, note that ordinary theta angles are encoded in $Z_{\text {class }}(\sigma, \mathfrak{m})$, in the

$$
\exp (-i \theta \operatorname{Tr} \mathfrak{m})
$$

factor. In this notation, in this term we interpret $\mathfrak{m}$ as a matrix in the Lie algebra of a Cartan torus 14 in the gauge group, and the trace is taken over that matrix.

Now, for a semisimple gauge group, that trace will always vanish, and indeed one does not expect ordinary theta angles in two dimensions in a semisimple gauge theory. However, that term implicitly tells us how to insert discrete theta angles in these partition functions - by adding a (nonvanishing) term to the (vanishing) theta angle term.

In the case of $S O(3)$, a nonzero discrete theta angle is encoded as a factor

$$
\exp (-i \pi \mathfrak{m})=(-)^{\mathfrak{m}}
$$

placed in the same location in the partition function expression as the ordinary theta angle term. In other words, for odd $\mathfrak{m}$, corresponding to an $\mathfrak{m}$ in $S O(3)$ 's cocharacter lattice but not $S U(2)$ 's, this is a sign. (Note that our notation is ambiguous: for ordinary theta angles, we interpret $\mathfrak{m}$ as a matrix, whereas for the discrete theta angle term, we interpret $\mathfrak{m}$ as an integer. As both are, morally, the same $\mathfrak{m}$, we will continue to use ambiguous notation, and trust the reader to disambiguate at need.)

We can now see, for $S U(2)$ gauge theories with center-invariant matter, how the decomposition conjecture is realized in partition functions. Specifically, for $S U(2)$, the partition

to refer to the lattice of weights of representations of a particular Lie group, whereas for others it is defined with respect to the Lie algebra only, independent of the Lie group. The former notion, defined in terms of representations of a particular Lie group, can be more invariantly characterized in terms of homomorphisms from the maximal torus of the gauge group to the circle, and so is often called the character lattice. The dual lattice we are interested in here is similarly known as the cocharacter lattice. We would like to thank T. Pantev for a useful discussion of this issue.

${ }^{14}$ In writing this, we are utilizing the fact that the cocharacter lattice can be interpreted as a subset of the Cartan torus. 
function (15) is a sum over only even $\mathfrak{m}$; for $S O(3)_{+}$, a sum over all integer $\mathfrak{m}$; for $S O(3)_{-}$, a sum over all integer $\mathfrak{m}$ but containing an extra $(-)^{\mathfrak{m}}$. Schematically, if we write 15

$$
Z\left(S O(3)_{+}\right)=\frac{1}{2} \sum_{\mathfrak{m} \in \mathbb{Z}} A(\mathfrak{m})
$$

for a function $A(\mathfrak{m})$ which encodes all the $\mathfrak{m}$-dependence, then

$$
Z\left(S O(3)_{-}\right)=\frac{1}{2} \sum_{\mathfrak{m} \in \mathbb{Z}}(-)^{\mathfrak{m}} A(\mathfrak{m})
$$

(for a theory with the same matter), and

$$
Z(S U(2))=\sum_{\mathfrak{m} \in 2 \mathbb{Z}} A(\mathfrak{m})=Z\left(S O(3)_{+}\right)+Z\left(S O(3)_{-}\right)
$$

(for an $S U(2)$ theory with the same matter). Thus, we see that exact $S^{2}$ partition functions of supersymmetric gauge $S U(2)$ theories with center-invariant $\sqrt{16}$ matter factor in precisely the form predicted by decomposition, providing a consistency check.

Before describing the general case, let us consider another example: $S U(3)$. The simple roots of $S U(3)$ can be represented as [44][section 7.2]

$$
\left(\frac{1}{2}, \frac{\sqrt{3}}{2}\right), \quad\left(\frac{1}{2},-\frac{\sqrt{3}}{2}\right)
$$

from which we derive that the cocharacter lattice for $S U(3) / \mathbb{Z}_{3}$ has elements of the form

$$
\left(2 m, \frac{2}{\sqrt{3}} n\right)
$$

15 This expression is valid for both the pure supersymmetric theory as well as a theory with matter: the difference between the two is encoded in the function $A(\mathfrak{m})$. We have included an overall factor of $(1 / 2)$ to reflect the change in size of the integration region - because $S U(2)$ has twice the volume of $S O(3)$, in principle one would expect that gauging $S O(3)$ should result in a path integral with a normalization of $1 / 2$ relative to gauging an $S U(2)$. That said, in quantum field theories not coupled to gravity, overall normalizations of the partition function are not meaningful, and can be absorbed into counterterms (see e.g. 43 , for a pertinent discussion). Thus, it might be better to characterize such choices as conventions. In that language, we have chosen convention-dependent factors to make the discussion more clear.

16 If the matter is not center-invariant, the partition function can still be written as a sum of two infinite series, but neither infinite series seems to be interpretable as the partition function of a gauge theory. In particular, the derivation of $Z_{\Phi}$ for a chiral superfield in representation $R$ explicitly assumes that $\rho(\mathfrak{m})$ is an integer for all weights $\rho$ and elements $\mathfrak{m}$ of the cocharacter lattice: for example, [41] [section 3.2] computes the pertinent operator determinant by expanding in a series of spin spherical harmonics of $\operatorname{spin}(1 / 2)|\rho(\mathfrak{m})|$, which is only sensible if $\rho(\mathfrak{m})$ is an integer. More generally, an infinite sum can be written in a variety of ways as a formal sum of other infinite sums; what is pertinent here is that the infinite sums have a physical meaning as partition functions of gauge theories, following the pattern predicted by decomposition. 
for $m, n \in \mathbb{Z}$. The weights of the fundamental can, in the same conventions, be represented as

$$
\left(\frac{1}{2}, \frac{\sqrt{3}}{6}\right), \quad\left(-\frac{1}{2}, \frac{\sqrt{3}}{6}\right), \quad\left(0,-\frac{\sqrt{3}}{3}\right)
$$

from which we deduce that the cocharacter lattice for $S U(3)$ has elements of the form

$$
\left(2 m, \frac{6}{\sqrt{3}} n\right) \text {. }
$$

Now, if we are given

$$
\mathfrak{m}=\left(2 m, \frac{2}{\sqrt{3}} n\right)
$$

in the cocharacter lattice of $S U(3) / \mathbb{Z}_{3}$, the $\mathbb{Z}_{3}$-valued analogue of the second Stiefel-Whiney class is determined by $n$ mod 3 . Thus, we could add a discrete theta angle to the partition function of an $S U(3) / \mathbb{Z}_{3}$ theory by adding a term

$$
\exp (-i \theta n)
$$

for $n$ determined by $\mathfrak{m}$ as above and $\theta \in\{0,2 \pi / 3,4 \pi / 3\}$. In other words, if $w$ denotes the integral of the $\mathbb{Z}_{3}$-valued analogue of the Stiefel-Whitney class, then in effect,

$$
w(\mathfrak{m})=n \bmod 3 .
$$

With that in hand, decomposition can be checked for $S U(3)$ theories in the same form as for $S U(2)$ theories. As before, we can write

$$
Z(S U(3))=\sum_{m \in \mathbb{Z}} \sum_{n \in 3 \mathbb{Z}} A(\mathfrak{m})
$$

for the $S^{2}$ partition function of an $S U(3)$ theory with center-invariant matter, where $\mathfrak{m}$ is determined by integers $m, n$ as above, and $A(\mathfrak{m})$ is defined for $\mathfrak{m}$ corresponding to arbitrary integer $m, n$. We can decompose this as

$$
\sum_{m \in \mathbb{Z}} \sum_{n \in 3 \mathbb{Z}} A(\mathfrak{m})=\frac{1}{3} \sum_{m \in \mathbb{Z}} \sum_{n \in \mathbb{Z}} A(\mathfrak{m})+\frac{1}{3} \sum_{m \in \mathbb{Z}} \sum_{n \in \mathbb{Z}} e^{-2 \pi i n / 3} A(\mathfrak{m})+\frac{1}{3} \sum_{m \in \mathbb{Z}} \sum_{n \in \mathbb{Z}} e^{-4 \pi i n / 3} A(\mathfrak{m}),
$$

which can then be interpreted as

$$
Z(S U(3))=Z\left(\left(S U(3) / \mathbb{Z}_{3}\right)_{0}\right)+Z\left(\left(S U(3) / \mathbb{Z}_{3}\right)_{1}\right)+Z\left(\left(S U(3) / \mathbb{Z}_{3}\right)_{2}\right),
$$

precisely in accord with the prediction of decomposition.

It is straightforward to generalize this to more general semisimple gauge groups $G$. In general, for semisimple $G$ with center $K$, if $M_{G}$ denotes the cocharacter lattice for $G$, then 
$M_{G} \subset M_{G / K}$ and $M_{G / K} / M_{G}$ has as many elements as $K$. In each case, to evaluate partition functions explicitly, one must represent the integral of the analogue of the second StiefelWhitney class as an invariant $w$ of the cocharacter lattice, and that invariant determines the pertinent discrete theta angle term in the partition function. That invariant $w$ is encoded in the relation 17 between the cocharacter lattices:

$$
1 \longrightarrow M_{G} \longrightarrow M_{G / K} \stackrel{w}{\longrightarrow} K \longrightarrow 1 .
$$

The decomposition statement is encoded in the fact that

$$
\frac{1}{|K|} \sum_{\mu \in \hat{K}} e^{i \mu(w(\mathfrak{m}))}
$$

is a projection operator that projects the lattice $M_{G / K}$ onto $M_{G}$, in other words, is the identity on $\mathfrak{m} \in M_{G / K}$ in the image of $M_{G}$, but vanishes otherwise.

It is now straightforward to check that the partition functions obey decomposition. For example, for $G$ semisimple and $K$ a (finite) subgroup of the center, decomposition in the form (4) can be checked as follows:

$$
\begin{aligned}
Z(G) & =\sum_{\mathfrak{m} \in M_{G}} A(\mathfrak{m}), \\
& =\frac{1}{|K|} \sum_{\lambda \in \hat{K}} \sum_{\mathfrak{m} \in M_{G / K}} e^{i \lambda(w(\mathfrak{m}))} A(\mathfrak{m}), \\
& =\sum_{\lambda \in \hat{K}} Z\left((G / K)_{\lambda}\right),
\end{aligned}
$$

where $M_{G}$ is the cocharacter lattice of $G$, and

$$
Z\left((G / K)_{\lambda}\right)=\frac{1}{|K|} \sum_{\mathfrak{m} \in M_{G / K}} e^{i \lambda(w(\mathfrak{m}))} A(\mathfrak{m}) .
$$

The same methods can also be applied to check decomposition in the more general form (5): for $G=\tilde{G} / K, G$ semisimple, $\tilde{G}$ simply-connected, $K$ a subgroup of the center of $\tilde{G}$, and $\lambda \in \hat{K}$,

$$
\begin{aligned}
Z\left((G)_{\lambda}\right) & =\sum_{\mathfrak{m} \in M_{G}} e^{i \lambda\left(w_{K}(\mathfrak{m})\right)} A(\mathfrak{m}), \\
& =\frac{1}{|C(G)|} \sum_{\mu \in \widehat{C(G)}} \sum_{\mathfrak{m} \in M_{G / C(G)}} e^{i(\lambda, \mu) w_{C(\tilde{G})}(\mathfrak{m})} A(\mathfrak{m}), \\
& =\sum_{\mu \in \widehat{C(G)}} Z\left((G / C(G))_{\lambda, \mu}\right) .
\end{aligned}
$$

\footnotetext{
${ }^{17}$ See appendix $\AA$ for a derivation.
} 
In the expression above, $w_{K}: M_{G} \rightarrow K\left(\right.$ with kernel $\left.M_{\tilde{G}}\right), w_{C(\tilde{G})}: M_{G / C(G)}\left(=M_{\tilde{G} / C(\tilde{G})}\right) \rightarrow$ $C(\tilde{G})$ with kernel $M_{\tilde{G}}$, and $w_{C(G)}: M_{G / C(G)} \rightarrow C(G)$ with kernel $M_{G}$. The projection operator has been modified slightly: we have replaced the factor

$$
e^{i \mu\left(w_{C(G)}(\mathfrak{m})\right)}
$$

(which reduces to 1 on the image of $M_{G}$ in $M_{G / C(G)}$ ) with

$$
e^{i(\lambda, \mu) w_{C(\tilde{G})}(\mathfrak{m})} .
$$

We can see that this reduces to the desired expression on $M_{G}$ as follows. Becauss $w_{C(G)}=$ $\alpha \circ w_{C(\tilde{G})}$ where

$$
1 \longrightarrow K \longrightarrow C(\tilde{G}) \stackrel{\alpha}{\longrightarrow} C(G) \longrightarrow 1,
$$

and the fact that the image of $M_{G}$ is in the kernel of $w_{C(G)}$, we see that the image of $M_{G}$ in $M_{\tilde{G} / C(\tilde{G})}=M_{G / C(G)}$ must lie in the image of $K$, hence from equation (6) and commutivity 19 of the square

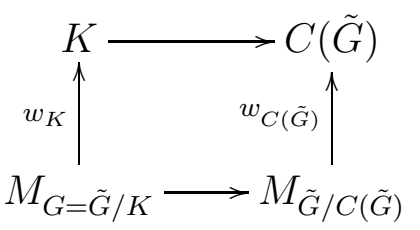

we find that

$$
e^{i(\lambda, \mu) w_{C(\tilde{G})}(\mathfrak{m})}=e^{i \lambda w_{K}(\mathfrak{m})}
$$

for $\mathfrak{m}$ in the image of $M_{G}$. Thus, our modified projection operator reduces to the desired form on $M_{G}$.

\section{Four dimensional theories}

In four dimensional theories, it is no longer the case that a gauge theory with a triviallyacting subgroup is physically equivalent to a gauge theory with a restriction on instantons. For example, in four dimensions, pure $N=1$ supersymmetric $S U(2)$ Yang-Mills theory does not violate cluster decomposition, and has the same number of vacua in the IR as pure $N=1$ supersymmetric $S O(3)$ Yang-Mills theory.

However, it is nonetheless intriguing to compare, for example, $S U(2)$ gauge theories to $S O(3)$ gauge theories with restrictions on instantons.

In this section, we will discuss four-dimensional gauge theories with restrictions on instantons, enforced by inserting projection operators into path integrals. These theories do not

\footnotetext{
${ }^{18}$ See appendix $\mathrm{A}$ for a derivation.

${ }^{19}$ See appendix $\AA$ for a derivation.
} 
obey cluster decomposition, but, at least for SCFT's, have been argued to obey an analogue of the decomposition conjecture [11][appendix A], so that cluster decomposition is violated in an extremely mild way. That said, unlike decomposition in two dimensions, there has been very little work done on decomposition in four dimensions, so part of the purpose of this section is to further illuminate decomposition in four dimensional theories. For simplicity, we will restrict to four-dimensional SCFT's, both because these were the theories considered in [11] [appendix A], and also so that we can reliably work in a weak coupling regime.

We will first examine dyon spectra in four-dimensional theories with restrictions on instanton sectors. Reducing the number of instantons changes the theta angle periodicity, hence one can in principle run into a consistency problem in theta angle charge rotations [45]. If the theory decomposes into a disjoint union of (ordinary) theories, a contradiction is averted. Furthermore, we will see that dyon spectra can be used to distinguish an $S U(2)$ gauge theory from an $S O(3)$ gauge theory with a restriction on instantons obeying an analogue of decomposition. We will also briefly examine Vafa-Witten topological field theory partition functions, as they can be used to provide simple examples of the four dimensional decomposition of [11][appendix A] in action.

\subsection{Dyon charge lattices}

Let us begin by examining dyon spectra in four dimensional theories with restrictions on instantons. Start with a four-dimensional gauge theory with conventions chosen such that $\theta$ is $2 \pi$-periodic. Restrict allowed instantons to those with instanton numbers divisible by some integer $k>0$. The restricted theory has a different $\theta$ periodicity: the theory is invariant under $\theta \equiv \theta+2 \pi / k$.

Because of how dyon charges depend upon the $\theta$ angle [45], this constrains dyon spectra. In the original theory, the physics and so the dyon spectrum 20 was invariant under $\theta \mapsto \theta+2 \pi$, but in the new theory, it must be invariant under $\theta \mapsto \theta+2 \pi / k$.

This would appear to pose a consistency problem for the theory. If, for example, we have a dyon of charge $\left(\lambda_{e}, \lambda_{m}\right)$, then under a $2 \pi / k$ rotation of $\theta$, the dyon charge would become [45] $\left(\lambda_{e}+\lambda_{m} / k, \lambda_{m}\right)$, which, except for special values of $k$, is unlikely to land somewhere in the original charge lattice.

Nevertheless, it is still possible for the charge lattice to be closed under such a rotation. One universal solution is if the dyon spectrum is a sum of copies of the dyon spectrum of the original theory, but with $\theta$ rotated in increments of $2 \pi / k$, as arises in a disjoint union of theories on the same spacetime. (This is a prediction of the conjectured analogue of

\footnotetext{
${ }^{20}$ Individual dyon charges rotate as described in [45, but the spectrum as a whole remains invariant.

21 The dyon charge formula, although it depends upon $\theta$, is a purely semiclassical result independent of instanton sector [45], and so it is not affected by the restriction on allowed instantons.
} 
the decomposition conjecture of [1] in four-dimensional theories [11].) Ordinarily locality as discussed in [27][section 1.1] would prohibit dyon spectra of this form; however, if we have a disjoint union of theories, so that dyons associated with different theories do not interact, then locality only applies within each separate sector, and so any contradiction is averted.

The solution above is not unique, and we will see that in special cases, other solutions are possible. One example we will study in detail will involve the relation between an $S O(3)$ gauge theory with a restriction on instantons, and an $S U(2)$ theory. We will see that the $S U(2)$ theory encodes a different solution to the corresponding problem in this context.

Let us consider a concrete example to make this proposal more precise. Consider an $S U(2)$ gauge theory with a restriction on instantons, restricted to instantons with instanton number divisible by 3 . The dyon spectrum of the original $S U(2)$ theory itself has the form [27]

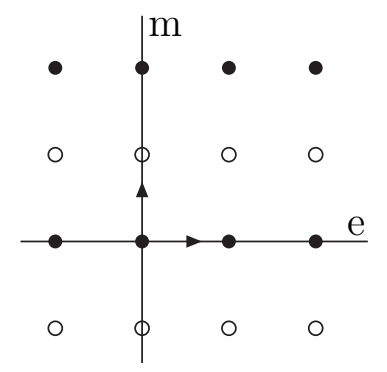

(Allowed electric charges are arbitrary integers; allowed magnetic charges are even integers.) Under $\theta \mapsto \theta+2 \pi / 3$, the charges rotate as $\left(\lambda_{e}, \lambda_{m}\right) \mapsto\left(\lambda_{e}+\lambda_{m} / 3, \lambda_{m}\right)$, and the resulting charge lattice has the form

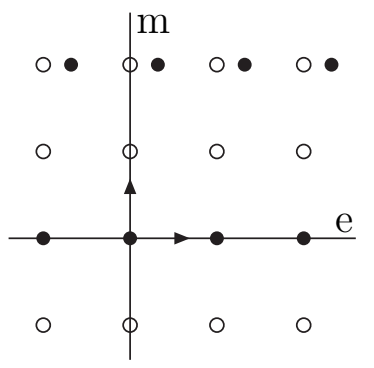

Here, the electric charges of dyons with $\lambda_{m}=2$ are shifted by $1 / 3$. Similarly, the electric charges of dyons with $\lambda_{m}=4$ would be shifted by $2 / 3$, and so forth.

As anticipated above, under this rotation, the dyon spectrum is not invariant. However, the physics should be invariant, as we are rotating the theta angle by its periodicity. As suggested above, one solution is that the correct dyon spectrum is determined by a set of dyons arising from variable $\theta$ angles. The resulting dyon spectrum is given by the sum of the two spectra above, plus another resulting from rotating $\theta \mapsto \theta+4 \pi / 3$, to get 


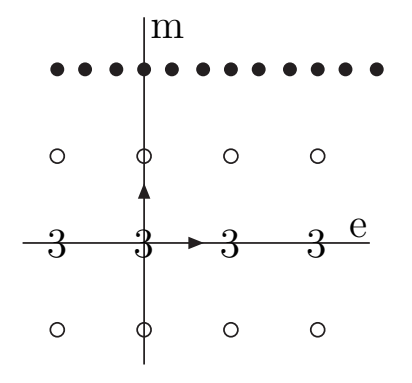

We have used "3"'s to indicate that there are three separate dyons with the same listed charges. As noted earlier, this dyon spectrum is only sensible if the theory decomposes into a disjoint union; our point is that such a decomposition gives a dyon spectrum compatible with theta angle dyon charge rotations.

Another example will be instructive. Let us compare $S U(2)$ to the $S O(3)_{ \pm}$gauge theories. These are very similar: all have the same perturbative description, for example, as the Lie algebras match. Furthermore, the $S U(2)$ instantons are a subset of the $S O(3)$ instantons, which suggests (incorrectly) that the $S U(2)$ theory is equivalent to an $S O(3)$ theory with a restriction on instantons. Let us work through this in detail, to see how the $S U(2)$ theory is distinguished from $S O(3)_{ \pm}$with a restriction on instantons.

Specifically, consider the four-dimensional $S O(3)_{-}$theory [27, 28]. Let us restrict the

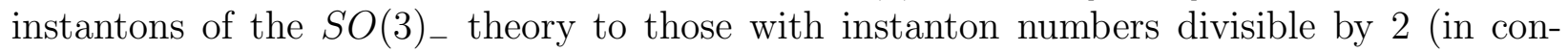

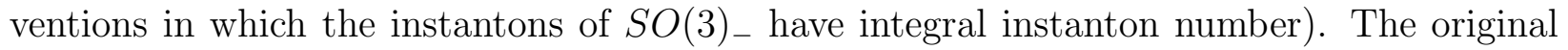
$S O(3)_{-}$theory is periodic under $\theta \mapsto \theta+4 \pi$; the new theory with the restriction on instanton numbers is periodic under $\theta \mapsto \theta+2 \pi$.

The prediction above is that in this restricted theory, the dyon spectrum is the sum of the dyons in two different theories:

$$
S O(3)_{-}^{\theta} \text { and } S O(3)_{-}^{\theta+2 \pi}=S O(3)_{+}^{\theta} .
$$

(Hand-in-hand, the physical theory must similarly decompose into a disjoint union.) In the figure below, we illustrate dyon charge multiplicities occuring in the resulting spectrum:

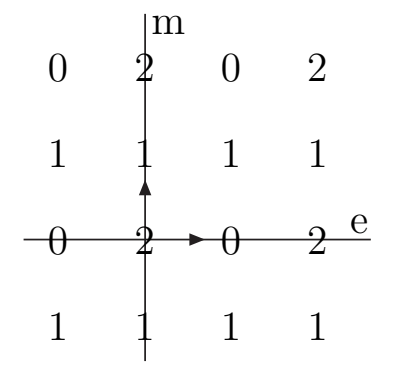

As a consistency check, under the rotation $\theta \mapsto \theta+2 \pi$, note that the lattice as a whole is 
invariant under the dyon charge rotation [45]

$$
\left(\lambda_{e}, \lambda_{m}\right) \mapsto\left(\lambda_{e}+\lambda_{m}, \lambda_{m}\right)
$$

For example, when $\lambda_{m}$ is even, the only allowed $\lambda_{e}$ 's are all even, and so they shift into one another. When $\lambda_{m}$ is odd, all $\lambda_{e}$ 's are permitted, and they shift into one another.

In this case, the solution above is not unique. Another dyon spectrum which is also closed under $\theta \mapsto \theta+2 \pi$ is of the form

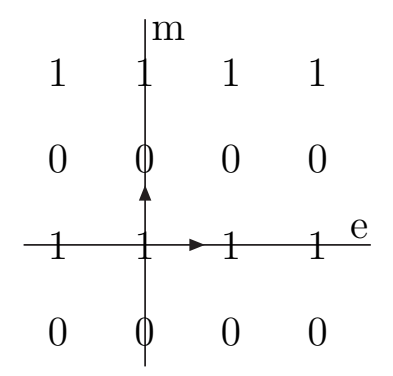

and is significantly different from the spectrum above. For example, in this spectrum we do not have multiple copies of any dyons; this theory appears to be "connected," loosely speaking. In fact, this is precisely the spectrum of the $S U(2)$ theory [27]. Other than the spectrum of dyons, however, the restricted $S O(3)_{-}$and $S U(2)$ theories are very similar: both have the same perturbative description, both have the same instantons, but the dyon spectrum in the two cases is significantly different.

\subsection{Vafa-Witten TFT partition functions}

As four dimensional decomposition has not been checked nearly as thoroughly as two dimensional cases, it is useful to study further examples. To that end, in this section we will discuss partition functions of the topological field theories of [46], arising from topologically-twisted four-dimensional $N=4$ theories, with a restriction on instantons. We shall see that those partition functions can be written as partition functions of disjoint unions of theories, in agreement the four-dimensional decomposition prediction of [11][appendix A]. In fact, the same arguments can be applied verbatim to other supersymmetric instanton computations, such as the Nekrasov partition function, as the partition functions have the same general form. For simplicity, we will only describe Vafa-Witten partition functions. (Of course, a thorough check of decomposition in this case would require more than comparing partition functions - we would need to also demonstrate that correlation functions factorize, for example, as has been shown in related two-dimensional theories. Our purpose here is merely to provide some evidence supporting the argument of [11][appendix A].) 
Briefly, the partition functions of these topological field theories are of the form [46]

$$
Z(q)=\sum_{n} a_{n} q^{n},
$$

where

$$
q=e^{2 \pi i \tau}=\exp \left(i \theta-\frac{8 \pi^{2}}{g^{2}}\right),
$$

and $a_{k}$ is proportional to the moduli space of $n$ instantons. Suppose, for example, that we consider the analogous theory defined by a restriction to instanton numbers divisible by $k$. Then the partition function of this new theory would be

$$
Z_{k}(q)=\sum_{n} a_{n k} q^{n k} .
$$

Note that this is the same as

$$
\sum_{n}\left(\frac{1}{k} \sum_{\alpha=0}^{k-1} e^{2 \pi i \alpha n / k}\right) a_{n} q^{n}=\frac{1}{k} \sum_{\alpha=0}^{k-1} \sum_{n} a_{n} q^{n} e^{2 \pi i \alpha n / k} .
$$

The effect of the insertion is to project onto instanton numbers divisible by $k$ : when $n$ is divisible by $k$, each factor in the $\alpha$ sum is 1 , otherwise the $\alpha$ sum is a sum over roots of unity, which vanishes. At the same time, writing the partition function for the theory with a restriction on instanton sectors, in this form,

$$
\begin{aligned}
Z_{k}(q) & =\frac{1}{k} \sum_{\alpha=0}^{k-1} \sum_{n} a_{n} q^{n} e^{2 \pi i \alpha n / k}=\frac{1}{k} \sum_{\alpha=0}^{k-1} \sum_{n} a_{n} \exp \left(n\left(i \theta+i 2 \pi \alpha / k-\frac{8 \pi^{2}}{g^{2}}\right)\right), \\
& =\frac{1}{k} \sum_{\alpha=0}^{k-1} Z\left(q_{\alpha}\right),
\end{aligned}
$$

where

$$
q_{\alpha}=\exp \left(i \theta+i 2 \pi \alpha / k-\frac{8 \pi^{2}}{g^{2}}\right),
$$

consistent with an interpretation as a disjoint union of theories with rotating $\theta$ angles, as predicted in [11].

\section{Dijkgraaf-Witten theory}

The recent paper [47] studied strings on gerbes, the same sorts of theories we have discussed in this paper, albeit described there in the language of QFT's coupled to TQFT's. The prototype for the TQFT coupling in [47] was given by Dijkgraaf-Witten theory [48]. Briefly, 
we can understand analogues of Dijkgraaf-Witten theory in $n$ dimensions, as defined by [47], directly in finite gauge theory, without the overhead of various abelian gauge theories. In such language, the theories of [47] can be described as an $n$-dimensional orbifold of a point with an analogue of discrete torsion turned on.

For example, in two dimensions with gauge group $G$, the partition function of the twodimensional version 22 of Dijkgraaf-Witten theory on $T^{2}$ is given by

$$
Z_{2}=\frac{1}{|G|} \sum_{g h=h g} \frac{\alpha(g, h)}{\alpha(h, g)}
$$

where $\alpha \in H^{2}(G, U(1))$ is a group cocycle (with trivial action on the coefficients). The ratio

$$
\frac{\alpha(g, h)}{\alpha(h, g)}
$$

is invariant under both coboundaries and $S L(2, \mathbb{Z})$.

In three dimensions, with gauge group $G$ the partition function of Dijkgraaf-Witten theory has a similar form, with phase factors now given by the analogues of discrete torsion for the $C$ field [49,50]. For example, on a three-torus, the partition function is given by

$$
Z_{3}=\frac{1}{|G|} \sum_{\text {commuting triples permutations } P} \alpha\left(g_{i_{1}}, g_{i_{2}}, g_{i_{3}}\right)^{ \pm P} .
$$

The alternating ratio of cocycles $\alpha \in H^{3}(G, U(1))$ can be shown to be both invariant under coboundaries and also invariant under $S L(3, \mathbb{Z})[48,49]$.

Similarly, in $d$ dimensions, for gauge group $G$ the analogue of Dijkgraaf-Witten theory has a similar form. For example, on a $d$-torus, the partition function is given by

$$
Z_{d}=\frac{1}{|G|} \sum_{\text {commuting pairs permutations } P} \alpha\left(g_{i_{1}}, g_{i_{2}}, \cdots, g_{i_{d}}\right)^{ \pm P},
$$

where $\alpha$ is a cocycle representing an element of $H^{d}(G, U(1))$, with trivial action on the coefficients. (It is straightforward to check that this expression is invariant under coboundaries.) (See also [51] for a derivation of such expressions from simplices in two dimensions, and [48] for an analogous derivation in three dimensions.)

Now, turning on discrete torsion in a trivially-acting subgroup of a gauge group in two dimensions modifies the decomposition conjecture, as observed in [1] [section 10]. In particular, in the presence of discrete torsion, decomposition may no longer take place at all.

22 The original reference [48] only considered three-dimensional theories, but glossing over occasional subtleties such as certain functoriality issues in low dimensions, analogues are straightforward to write down in any dimension, as indeed [47] do. 
A prototypical example is provided by the two-dimensional orbifold $\left[X /\left(\mathbb{Z}_{2} \times \mathbb{Z}_{2}\right)\right]$, where $X$ is a manifold (defining a nonlinear sigma model) and the first $\mathbb{Z}_{2}$ acts trivially on $X$. It was argued in [1] [section 10.1] that in this case, if discrete torsion is turned on, the theory on $\left[X /\left(\mathbb{Z}_{2} \times \mathbb{Z}_{2}\right)\right]$ is equivalent to a sigma model on one copy of $X$ - a 2-fold cover of $\left[X / \mathbb{Z}_{2}\right]$. More generally, for the two-dimensional orbifold $\left[X /\left(\mathbb{Z}_{k} \times \mathbb{Z}_{k}\right)\right]$ where the first $\mathbb{Z}_{k}$ acts trivially, for any nontrivial discrete torsion, this theory is equivalent 23 [1][section 10.2] to a sigma model on one copy of $X$, a $k$-fold cover of $\left[X / \mathbb{Z}_{k}\right]$. (The fastest way to see this is to compute the genus one worldsheet partition function - because of the discrete torsion phase factors, all contributions from twisted sectors cancel out, leaving just the partition function of an ordinary nonlinear sigma model on $X$.)

\section{Conclusions}

In this paper we have discussed 'decomposition' in two and four dimensions. In two dimensions, it is a long established result that in orbifolds and abelian gauge theories, if a finite subgroup leaves matter invariant, then the theory 'decomposes' into a disjoint union of theories. As there is no gauge dynamics in two dimensions, one therefore expects closely related phenomena in two-dimensional nonabelian gauge theories. We gave a general conjecture for its formulation, and demonstrated that both nonsupersymmetric pure two-dimensional Yang-Mills and supersymmetric theories in two dimensions obey the general principle. In particular, this allowed us to derive a decomposition result for pure two-dimensional nonsupersymmetric Yang-Mills.

In four dimensions, existence of a trivially-acting finite subgroup is no longer equivalent to a restriction on instantons. We discussed how these can be distinguished using dyon spectra, and also discussed partition function constructions in four-dimensional topological field theories with restrictions on instantons that mirror analogous two-dimensional constructions.

There are a number of other directions to pursue. For one example, it is well-known that two-dimensional Yang-Mills can also be formulated as a string theory (see e.g. [35, 54] for a complete description of that string theory). It would be interesting to understand how that string theory also decomposes.

For another example, zero-area limits of partition functions of $q$-deformed pure twodimensional Yang-Mills are now understood to compute some indices of four-dimensional superconformal field theories, see e.g. [55, 56]. It would be interesting to understand the implications of the decomposition conjecture described here for four-dimensional supercon-

${ }^{23}$ In passing, we should mention that there is also related work in the math literature, albeit describing a different duality. Specifically, the paper 52 gives a mathematical proposal for understanding decomposition with discrete torsion turned on in the fashion indicated here. However, in this example, the duality described in $\left[52\right.$ predicts $\left[53 . k\right.$ copies of $\left[X / \mathbb{Z}_{k}\right]$ rather than one copy of $X$. 
formal indices. Perhaps, although full four dimensional theories do not obey the same decomposition (unless one enforces a strong instanton restriction), the indices decompose.

Yet another direction to pursue is decomposition in three dimensions. Do three-dimensional theories with trivially-acting finite groups decompose, or only if one imposes a further restriction on instantons? Such questions would be interesting to understand.

\section{Acknowledgements}

We would like to thank R. Donagi, S. Hellerman, B. Jia, S. Katz, T. Pantev, Y. Tachikawa, X. Tang, and H.-H. Tseng for useful discussions. E.S. was partially supported by NSF grant PHY-1068725.

\section{A Miscellaneous cocharacter lattice relations}

In this appendix we shall derive a few relations between cocharacter lattices that are used in the text.

First, for semisimple $G$ with $K$ a finite subgroup of the center, if $M_{G}$ denotes the cocharacter lattice for $G$, then we shall show that

$$
1 \longrightarrow M_{G} \longrightarrow M_{G / K} \stackrel{w}{\longrightarrow} K \longrightarrow 1 \text {. }
$$

This relation can be derived as follows [57]. Begin with the short exact sequence

$$
1 \longrightarrow K \longrightarrow T_{G} \longrightarrow T_{G / K} \longrightarrow 1
$$

and apply the functor $\operatorname{Hom}(U(1),-)$. The result is

$$
1 \longrightarrow M_{G} \longrightarrow M_{G / K} \longrightarrow \operatorname{Ext}^{1}(U(1), K) \longrightarrow 1 \text {. }
$$

To compute Ext ${ }^{1}$ above, apply $\operatorname{Hom}(-, K)$ to

$$
0 \longrightarrow \mathbb{Z} \longrightarrow \mathbb{R} \longrightarrow U(1) \longrightarrow 1
$$

to get

$1 \longrightarrow \operatorname{Hom}(U(1), K) \longrightarrow \operatorname{Hom}(\mathbb{R}, K) \longrightarrow \operatorname{Hom}(\mathbb{Z}, K) \longrightarrow \operatorname{Ext}^{1}(U(1), K) \longrightarrow \operatorname{Ext}^{1}(\mathbb{R}, K)$

Now,

$$
\operatorname{Hom}(U(1), K)=1=\operatorname{Hom}(\mathbb{R}, K)
$$


since $U(1), \mathbb{R}$ are divisible groups and $K$ is torsion, and similarly $\operatorname{Ext}^{1}(\mathbb{R}, K)=0$ since $\mathbb{R}$ is torsion-free and $K$ is torsion. Thus,

$$
\operatorname{Ext}^{1}(U(1), K) \cong \operatorname{Hom}(\mathbb{Z}, K)=K
$$

instead of $\hat{K}$. We would like to thank T. Pantev for sharing this computation. We will denote the map $w: M_{G / K} \rightarrow K$ by $w_{K}$.

Next, define the map $\alpha: C(\tilde{G}) \rightarrow C(G)$, where $C(G)$ denotes the center of $G$, as the second nontrivial map in the sequence

$$
1 \longrightarrow K \longrightarrow C(\tilde{G}) \stackrel{\alpha}{\longrightarrow} C(G) \longrightarrow 1 \text {. }
$$

We shall show that the following diagram commutes:

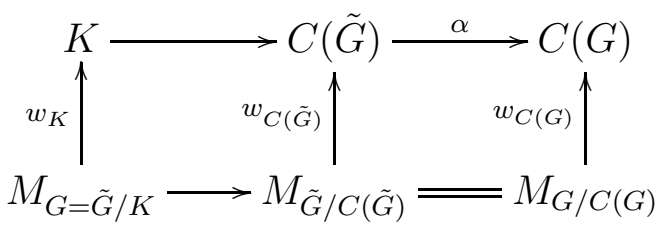

To show this, begin with the diagram

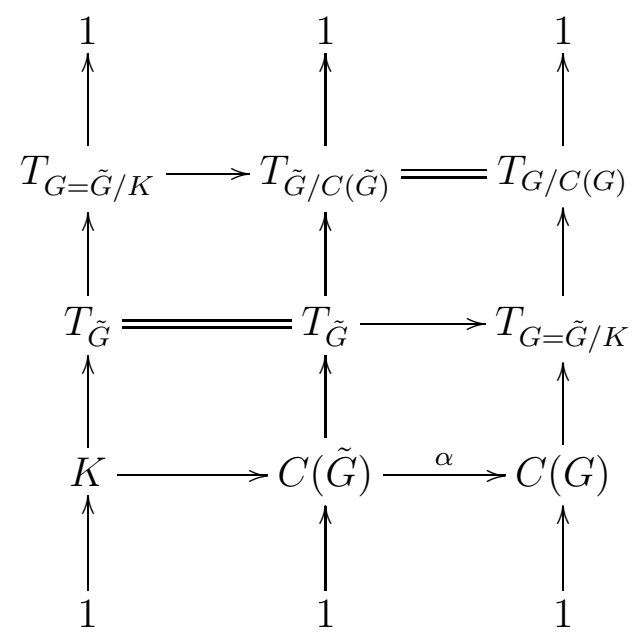

This commutes, hence the diagram obtained by applying $\operatorname{Hom}(U(1),-)$, namely

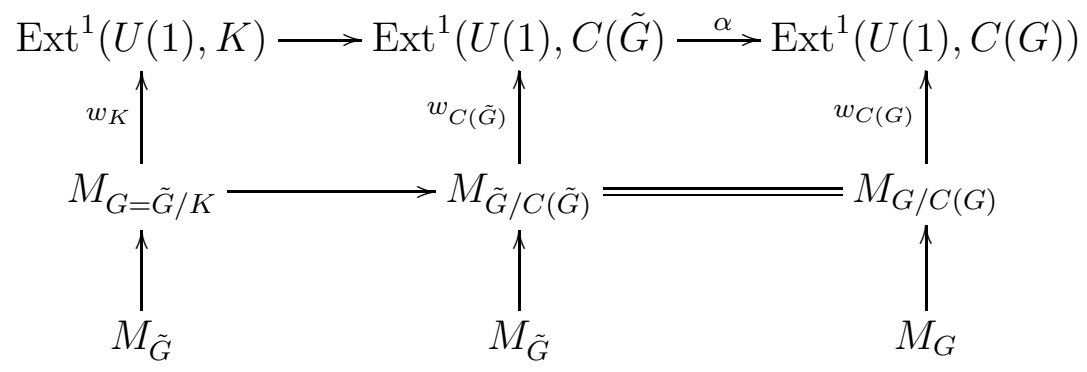

also commutes, which is the desired result. 


\section{References}

[1] S. Hellerman, A. Henriques, T. Pantev, E. Sharpe, M. Ando, "Cluster decomposition, T-duality, and gerby CFT's," Adv. Theor. Math. Phys. 11 (2007) 751-818, hep-th/0606034.

[2] A. Gadde, S. Gukov, P. Putrov, "(0,2) trialities," JHEP 1403 (2014) 076, arXiv: 1310.0818.

[3] D. Kutasov, J. Lin, "(0,2) dynamics from four dimensions," arXiv: 1310.6032.

[4] B. Jia, E. Sharpe, R. Wu, "Notes on nonabelian $(0,2)$ theories and dualities," arXiv: 1401.1511.

[5] D. Kutasov, J. Lin, “(0,2) ADE models from four dimensions," arXiv: 1401.5558.

[6] N. Bobev, K. Pilch, O. Vasilakis, "(0,2) SCFT's from the Leigh-Strassler fixed point," arXiv: 1403.7131.

[7] T. Pantev, E. Sharpe, "Notes on gauging noneffective group actions," hep-th/0502027.

[8] T. Pantev, E. Sharpe, "String compactifications on Calabi-Yau stacks," Nucl. Phys. B733 (2006) 233-296, hep-th/0502044.

[9] T. Pantev, E. Sharpe, "GLSM's for gerbes (and other toric stacks)," Adv. Theor. Math. Phys. 10 (2006) 77-121, hep-th/0502053.

[10] A. Caldararu, J. Distler, S. Hellerman, T. Pantev, E. Sharpe, "Non-birational twisted derived equivalences in abelian GLSMs," Comm. Math. Phys. 294 (2010) 605-645, arXiv: 0709.3855.

[11] S. Hellerman, E. Sharpe, "Sums over topological sectors and quantization of FayetIliopoulos parameters," Adv. Theor. Math. Phys. 15 (2011) 1141-1199, arXiv: 1012.5999.

[12] M. Berasaluce-González, M. Montero, A. Retolaza, A. M. Uranga, "Discrete gauge symmetries from (closed string) tachyon condensation," arXiv: 1305.6788.

[13] N. Addington, E. Segal, E. Sharpe, "D-brane probes, branched double covers, and noncommutative resolutions," arXiv: 1211.2446.

[14] J. Halverson, V. Kumar, D. Morrison, "New methods for characterizing phases of 2d supersymmetric gauge theories," arXiv: 1305.3278.

[15] E. Sharpe, "A few Ricci-flat stacks as phases of exotic GLSM's," Phys. Lett. B726 (2013) 390-395, arXiv: 1306.5440. 
[16] K. Hori, "Duality in two-dimensional $(2,2)$ supersymmetric nonabelian gauge theories," JHEP 1310 (2013) 121, arXiv: 1104.2853.

[17] L. Anderson, B. Jia, R. Manion, B. Ovrut, E. Sharpe, "General aspects of heterotic string compactifications on stacks and gerbes," arXiv: 1307.2269.

[18] E. Sharpe, "Derived categories and stacks in physics," contribution to the proceedings of the ESI research conference on homological mirror symmetry (Vienna, Austria, June 2006), arXiv: hep-th/0608056.

[19] E. Sharpe, "Landau-Ginzburg models, gerbes, and Kuznetsov's homological projective duality," to appear in the proceedings of Topology, $\mathbf{C}^{*}$ algebras, string duality (Texas Christian University, May 18-22, 2009).

[20] E. Sharpe, "GLSM's, gerbes, and Kuznetsov's homological projective duality," contribution to the proceedings of Quantum theory and symmetries 6, arXiv: 1004.5388 .

[21] E. Andreini, Y. Jiang, H.-H. Tseng, "On Gromov-Witten theory of root gerbes," arXiv: 0812.4477.

[22] E. Andreini, Y. Jiang, H.-H. Tseng, "Gromov-Witten theory of product stacks," arXiv: 0905.2258.

[23] E. Andreini, Y. Jiang, H.-H. Tseng, "Gromov-Witten theory of etale gerbes, i: root gerbes," arXiv: 0907.2087.

[24] H.-H. Tseng, "On degree zero elliptic orbifold Gromov-Witten invariants," Int. Math. Res. Not. IMRN 2011 2444-2468, arXiv: 0912.3580.

[25] A. Gholampour, H.-H. Tseng, "On Donaldson-Thomas invariants of threefold stacks and gerbes," Proc. Amer. Math. Soc. 141 (2013) 191-203, arXiv: 1001.0435.

[26] X. Tang, H.-H. Tseng, "Duality theorems of étale gerbes on orbifolds," Adv. Math. 250 (2014) 496-569, arXiv: 1004.1376.

[27] O. Aharony, N. Seiberg, Y. Tachikawa, "Reading between the lines of four-dimensional gauge theories," arXiv: 1305.0318.

[28] D. Gaiotto, G. Moore, A. Neitzke, "Framed BPS states," arXiv: 1006.0146.

[29] Y. Tachikawa, "On the 6d origin of discrete additional data of 4d gauge theories," arXiv: 1309.0697.

[30] K. Hori, "On global aspects of gauged Wess-Zumino-Witten model," hep-th/9402019.

[31] K. Hori, "Global aspects of gauged Wess-Zumino-Witten models," hep-th/9411134. 
[32] A. Migdal, "Recursion relations in gauge theories," Sov. Phys. JETP 42 413-418, (Zh. Eksp. Teor. Fiz. 69 (1975) 810-822).

[33] B. Rusakov, "Loop averages and partition functions in $U(N)$ gauge theory on twodimensional manifolds," Mod. Phys. Lett. A5 (1990) 693-703.

[34] D. Gross, W. Taylor, "Two-dimensional QCD is a string theory," hep-th/9301068.

[35] S. Cordes, G. Moore, S. Ramgoolam, "Lectures on 2d Yang-Mills theory, equivariant cohomology, and topological field theories," hep-th/9411210.

[36] E. Witten, "On quantum gauge theories in two dimensions," Comm. Math. Phys. 141 (1991) 153-209.

[37] M. Blau, G. Thompson, "Quantum Yang-Mills theory on arbitrary surfaces," Int. J. Mod. Phys. A7 (1992) 3781-3806.

[38] C. Klincik, "The formulae of Kontsevich and Verlinde from the perspective of the Drinfeld double," Comm. Math. Phys. 217 (2001) 203-228, hep-th/9911239.

[39] M. Blau, G. Thompson, "Lectures on 2d gauge theories: topological aspects and path integral techniques," hep-th/9310144.

[40] E. Katz, G. Tavares, Y. Xu, "Solving 2d QCD with an adjoint fermion analytically," arXiv: 1308.4980.

[41] F. Benini, S. Cremonesi, "Partition functions of $\mathrm{N}=(2,2)$ gauge theories on $S^{2}$ and vortices," arXiv: 1206.2356.

[42] N. Doroud, J. Gomis, B. Le Floch, S. Lee, "Exact results in $\mathrm{D}=2$ supersymmetric gauge theories," JHEP 1305 (2013) 093, arXiv: 1206.2606.

[43] E. Gerchkovitz, J. Gomis, Z. Komargodski, "Sphere partition functions and the Zamolodchikov metric," arXiv: 1405.7271.

[44] H. Georgi, Lie algebras in particle physics, second edition, Perseus Books, Reading, Massachusetts, 1999.

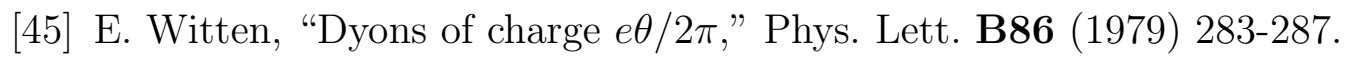

[46] C. Vafa, E. Witten, "A strong coupling test of S-duality," Nucl. Phys. B431 (1994) 3-77, hep-th/9408074.

[47] A. Kapustin, N. Seiberg, "Coupling a QFT to a TQFT and duality," arXiv: 1401.0740 .

[48] R. Dijkgraaf, E. Witten, "Topological gauge theories and group cohomology," Comm. Math. Phys. 129 (1990) 393-429. 
[49] E. Sharpe, "Analogues of discrete torsion for the M theory three form," Phys. Rev. D68 (2003) 126004, hep-th/0008170.

[50] E. Sharpe, "Recent developments in discrete torsion," Phys. Lett. B498 (2001) 104110, hep-th/0008191.

[51] P. Aspinwall, "A note on the equivalence of Vafa's and Douglas's picture of discrete torsion," JHEP 0012 (2000) 029, hep-th/0009045.

[52] I. Shapiro, X. Tang, H.-H. Tseng, "On the relative dual of an $S^{1}$-gerbe over an orbifold," arXiv: 1312.7316.

[53] X. Tang, H.-H. Tseng, private communication.

[54] S. Cordes, G. Moore, S. Ramgoolam, "Large N 2d Yang-Mills theory and topological string theory," Comm. Math. Phys. 185 (1997) 543-619, hep-th/9402107.

[55] A. Gadde, L. Rastelli, S. Razamat, W. Yan, "The 4d superconformal index from $q$ deformed 2d Yang-Mills," Phys. Rev. Lett. 106 (2011) 241602, arXiv: 1104.3850.

[56] A. Gadde, L. Rastelli, S. Razamat, W. Yan, "Gauge theories and Macdonald polynomials," Comm. Math. Phys. 319 (2013) 147-193, arXiv: 1110.3740.

[57] T. Pantev, private communication. 